\title{
Spectroscopy of early-type star candidates in M 33 and NGC 6822. II
}

\author{
G. Catanzaro ${ }^{1,2}$, L. Bianchi ${ }^{2}$, S. Scuderi ${ }^{1}$, and A. Manchado ${ }^{3,4}$ \\ 1 INAF - Catania Astrophysical Observatory, via S. Sofia n. 78, 95123, Catania, Italy \\ e-mail: scuderi@ct.astro.it \\ 2 Center for Astrophysical Sciences, The Johns Hopkins University, 3400 N. Charles St., Baltimore, MD 21218-2695, USA \\ e-mail: bianchi@pha.jhu.edu \\ 3 Instituto de Astrofisica de Canarias, via Láctea, 38200 La Laguna, Tenerife, Spain \\ e-mail: amt@ll.iac.es \\ ${ }^{4}$ Consejo Superior de Investigaciones Cientificas, Spain
}

Received 18 September 2002 / Accepted 5 March 2003

\begin{abstract}
We present ground-based spectra of early-type star candidates in the Local Group galaxies M 33 and NGC 6822 taken at the $4.2 \mathrm{~m}$ William Herschel Telescope (La Palma, Spain). Photospheric parameters $T_{\text {eff }}$ and $\log g$ were derived by LTE modeling of the Balmer lines. When possible, mass-loss rates were calculated from the $\mathrm{H}_{\alpha}$ profiles computing emission and scattering in the wind with the method of Scuderi et al. (1992). Five hot star candidates were found in M 33, out of seven stars analyzed, and two out of three in NGC 6822.
\end{abstract}

Key words. galaxies: individual: M33, NGC 6822 - stars: early type - stars: fundamental parameters - stars: atmospheres stars: mass loss

\section{Introduction}

This paper is part of our on-going effort (see Bianchi et al. 2001a,b) to characterize the massive star content of the galaxies of the Local Group. Aim of the program is to measure photospheric and wind parameters of hot massive stars through the analysis of photometric and spectroscopic measurements from ground based and HST data. These parameters will be compared with stars of similar spectral type but belonging to different galaxies in order to establish correlations with the global properties (i.e. metallicity or star formation history) of the parent galaxy. This work presents new spectroscopy for a sample of stars in OB associations of two of these galaxies: M 33 and NGC 6822.

The metal content of M 33 is similar to that measured in the Large Magellanic Cloud (LMC) (Vilchez et al. 1988; Russell \& Dopita 1990). The metallicity of NGC 6822 is lower than that of M33, being intermediate between that of the Small Magellanic Cloud (SMC) and the LMC (Pagel et al. 1980; Muschielok et al. 1999) with indications of extremely low metallicity in some regions (Chandar et al. 2000).

Send offprint requests to: G. Catanzaro, e-mail: gca@ct . astro.it

* Based on observations collected at the William Herschel Telescope, operated on the island of La Palma by the Royal Greenwich Observatory in the Spanish Observatorio del Roque de Los Muchachos of the Instituto de Astrofisica de Canarias.
We analyzed spectroscopic data for eight objects (seven stars and the nucleus) in M 33 and three stars in NGC 6822. For all the stars we derived $T_{\text {eff }}$ and $\log g$. Furthermore, for those stars showing clear evidence of stellar emission in $\mathrm{H}_{\alpha}$, we computed the mass loss rate. We also present a spectrum of M33's nuclear region.

\section{Observations and data reduction}

Long slit spectra were obtained with the ISIS spectrograph mounted on the $4.2 \mathrm{~m}$ William Herschel Telescope at the Observatorio del Roque de los Muchachos (La Palma, Spain) in August 1996. ISIS is a double beam spectrograph that uses dichroic slides to permit simultaneous observations in both the blue and the red arm. The ISIS blue arm was equipped with the TEK1 CCD, and the red arm with the TEK2 CCD; both CCDs had $1024 \times 1024$ pixels with a pixel size of $24 \mu \mathrm{m}$. The slit was 1.0 arcsec wide and 152 arcsec long, the plate scale was $\approx 0.38 \mathrm{arcsec} / \mathrm{pxl}$. Our spectra cover the regions from $4200 \AA$ to $5000 \AA$ (blue arm) and from $6200 \AA$ to $7000 \AA$ (red arm). Telluric lines show that the actual resolving power was $R \approx 5000$ and almost constant over the whole spectral range. The stars presented in this paper were included serendipitously in the long slits centered on targets presented elsewhere (Bianchi et al. 2003). The $S / N$ ratio varies widely in our spectra, with a maximum value of 50 , because the exposure time was optimized for the principal target of each field. 
We used in our analysis only portions of spectra with $S / N>$ 15 . Nevertheless, the $S / N$ combined with the moderate resolving power allowed us to perform a spectroscopic analysis for some of the serendipitous objects in order to obtain their physical properties.

Because of the limited $S / N$ ratio, we focused our attention on two spectral regions: $a$ ) a blue range from $4300 \AA$ to $4900 \AA$, containing $\mathrm{H}_{\gamma}$ and $\mathrm{H}_{\beta}$, $\mathrm{HeI} \lambda \lambda$ 4387.929, 4471.473, 4713.139 ̊, HeII $\lambda \lambda 4685.698,4713.273 \AA$, and some metallic lines: MgII 4481.150, NII 4641.028 and OII $4650.103 \AA$, and b) a red range, centered on $\mathrm{H}_{\alpha}$ and about $200 \AA$ wide.

The stellar spectra, calibrated in wavelength and with the stellar continuum normalized to a unity level, were obtained using standard data reduction procedures for spectroscopic observations within the NOAO/IRAF package. As several of our program stars lie in HII regions, extended nebular emission is also present. In order to correct for the sky contribution, we traced a sky order as close as possible to the star and as wide as the stellar trace. We then extracted the sky spectrum and subtracted it from the stellar order. However, given the spatial resolution of the instrument and the distance of the sources, it was not always possible to remove entirely the nebular emission from the stellar spectra. Consequently, the $\mathrm{H}_{\alpha}$ line often suffers from residual contamination.

\section{Data analysis}

In this study we follow a procedure similar to our previous work (Bianchi et al. 2001a, Paper I) to estimate the stellar parameters.

\subsection{Effective temperature and gravity}

The effective temperature, $T_{\text {eff }}$, and gravity, $\log g$, of the program stars were derived comparing the Balmer lines $\mathrm{H}_{\gamma}$ and $\mathrm{H}_{\beta}$ with synthetic spectra.

The synthetic spectra have been computed with the Kurucz code through the following steps: a) first, we calculated LTE model atmospheres for different values of $T_{\text {eff }}$ and $\log g$ using ATLAS9 (Kurucz 1993). This code takes into account the metal opacity using Opacity Distribution Functions for multiples of the solar metallicity. We used metallicity values of $Z=0.01$ for objects in M33 and $Z=0.002$ for objects in NGC 6822; b) synthetic spectra of the photospheric lines were calculated at high resolution with SYNTHE (Kurucz \& Avrett 1981) and c) convolved with a Gaussian profile to match the actual resolution of the data. As the resolution and the $S / N$ are too low to allow an estimate of the rotational velocity of the stars, we used the typical average value observed for their presumed spectral types of $v \sin i=150 \mathrm{~km} \mathrm{~s}^{-1}$ (Gray 1976).

The values of $T_{\text {eff }}$ and $\log g$ were determined by the best match of the model to the observed $\mathrm{H}_{\gamma}$ and $\mathrm{H}_{\beta}$. The temperature was constrained mostly from the depth of the line, and the gravity from the line wings. For those stars showing strong stellar wind emission in $\mathrm{H}_{\alpha}$, we included in our fitting procedure the effects of mass loss on the Balmer line profiles, thus deriving also the mass loss rate (see Sect. 3.2).

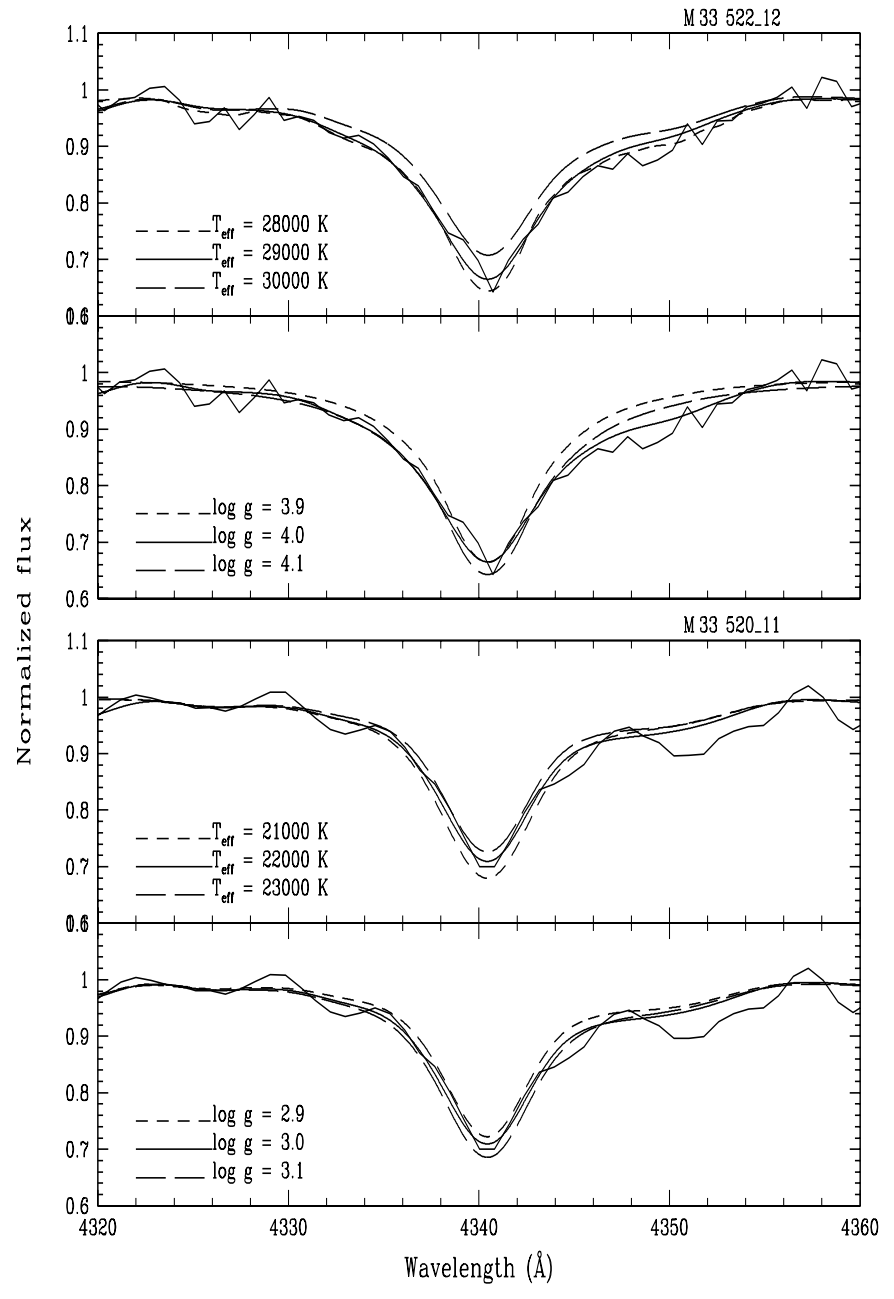

Fig. 1. Illustration of error estimates. The $\mathrm{H} \gamma$ profile of M 33 520_11 and M 33 522_12 is shown with the adopted models $\left(T_{\text {eff }}=22000 \mathrm{~K}\right.$ and $\log g=3.0$, and $T_{\text {eff }}=29000 \mathrm{~K}$ and $\log g=4.0$, bold line in both panels). For each star, the upper panel shows the variation of the profile with temperature, the dotted lines represent models with the same gravity and $T_{\text {eff }}=T_{\text {eff }}^{\text {adopted }} \pm 1000 \mathrm{~K}$. The bottom panel shows the variation with gravity, the dotted lines represent models with the same $T_{\text {eff }}$ and $\log g=\log g^{\text {adopted }} \pm 0.1$.

As an example of how the stellar parameters and their uncertainties are derived, we show in Fig. 1 the region around $\mathrm{H} \gamma$ for the stars M 33520_11 ( $T_{\text {eff }}=22000 \mathrm{~K}$ and $\log g=3.0$, bottom panel) and M $33522 \_12$ ( $T_{\text {eff }}=29000 \mathrm{~K}$ and $\log g=4.0$, upper panel). The solid smooth line is a model calculated with the adopted parameters. Uncertainties of $\Delta T_{\text {eff }}= \pm 1000 \mathrm{~K}$ and of $\Delta \log g= \pm 0.1$ dex are shown (dashed line models in Fig. 1).

Figure 1 illustrates the sensitivity of the models to the two main parameters $T_{\text {eff }}$ and $\log g$. Another source of uncertainty is the signal-to-noise ratio $(S / N)$ of the data. The $S / N$ affects the choice of the continuum level and consequently the depth and the shape of the line. To take in to account these effects on both $\Delta T_{\text {eff }}$ and $\Delta \log g$, we scaled the error derived from the model fit for the $S / N$ of each individual spectrum.

The value of the metallicity does not affect the hydrogen and helium lines, while its effect on other lines is not negligible. In Fig. 2 we show the spectral region between 


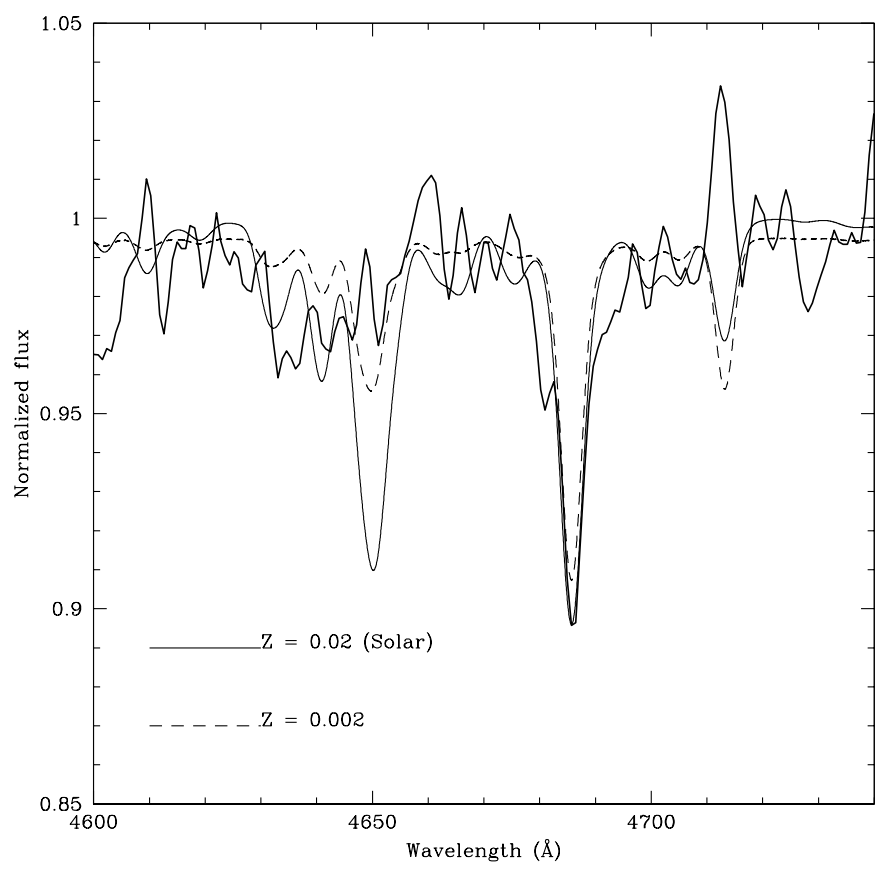

Fig. 2. Effects of metallicity. The observed (thick line) region between $4600 \AA$ and $4740 \AA$ A in NGC 6822 496_1 is compared with two models with different metallicities (solar and $Z=0.002$ ). The solar model shows a CIII $\lambda 4650.246 \AA$ line not present in the observed spectrum compatibly with the $S / N$ of this region. This line is strongly reduced in the low $Z$ model. The influence of the metallicity is instead negligible on the HeII $\lambda 4686 \AA$ line.

$4600 \AA$ and $4740 \AA$ of the star NGC 68224961 . The model calculated with solar metallicity (solid line) shows a strong CIII $\lambda 4650.246$ line and the HeII $\lambda 4685.698$ line. The helium line fits well the data while the carbon line is not present in the spectrum, due to the low metal content of NGC 6822. A synthetic spectrum computed with $\mathrm{Z}$ lower by 1 dex (dotted line) gives a better match of the metal line without degrading the fit of the HeII line. A precise metallicity determination was not attempted given the limited data quality as explained above.

As our analysis is based on LTE models, we investigated its limitations by assessing where non-LTE effects become non negligible. We compared our LTE models with non-LTE models obtained using the TLUSTY (Hubeny \& Lanz 1995) code to calculate the atmosphere model and SYNSPEC to calculate the synthetic spectra. Figure 3 shows a comparison between LTE and non-LTE $\mathrm{H}_{\gamma}, \mathrm{H}_{\beta}$ and $\mathrm{H}_{\alpha}$ profiles for $T_{\text {eff }}=30000 \mathrm{~K}$ and $\log g=4.0$. The theoretical profiles were convolved with a rotational profile and degraded to take into account the spectral resolution and a typical $S / N$ (which is $\approx 30$ ). The top panel of each box shows the ratio between the two profiles, which for these atmospheric parameters is lower than $10 \%$. At this temperature non-LTE effects become significant, and rapidly increase at higher temperatures.

For two objects, M $33522 \_12$ and M 33 522 9, our LTE analysis indicates $T_{\text {eff }}$ around $30000 \mathrm{~K}$. The uncertainties listed in Table 1 include an additional $10 \%$ due to the LTE approximation. For the two NGC 6822 objects hotter than $30000 \mathrm{~K}$ the contamination by nebular emission makes the temperature determination very uncertain with no advantage in a non-LTE

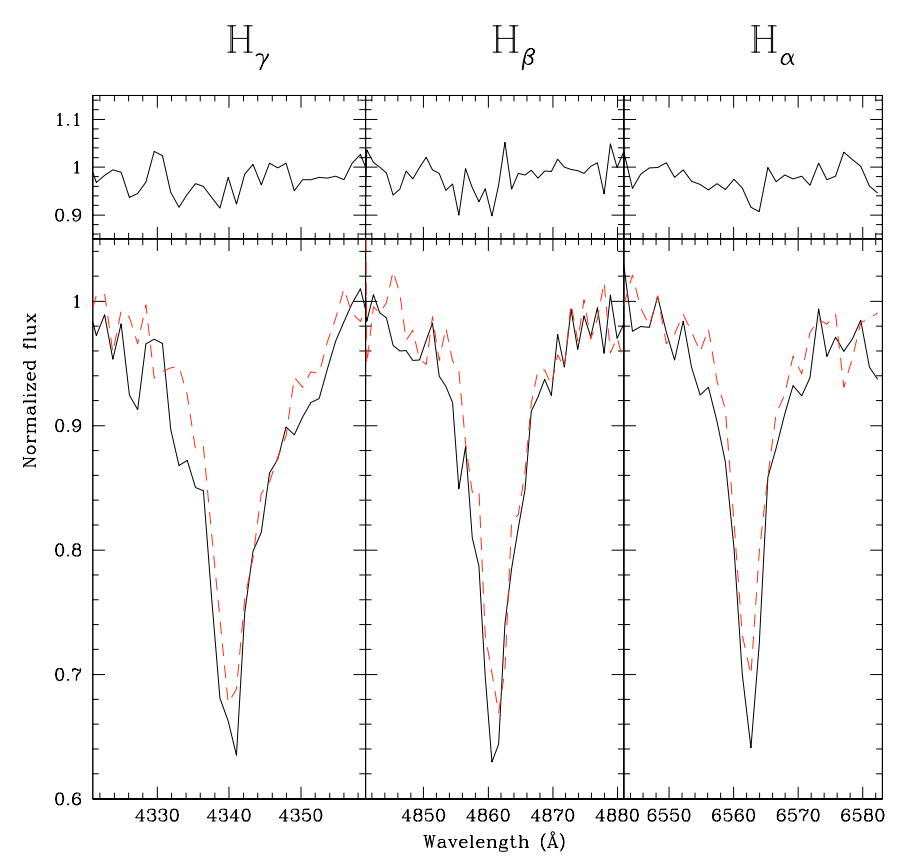

Fig. 3. Comparison between LTE versus non-LTE profiles of the Balmer lines $\mathrm{H}_{\gamma}, \mathrm{H}_{\beta}$ and $\mathrm{H}_{\alpha}$ for $T_{\text {eff }}=30000 \mathrm{~K}$ and $\log g=4.0$. LTE profiles (dashed curves) have been calculated with SYNTHE (Kurucz \& Avrett 1981) applied to an atmosphere model computed using ATLAS9 (Kurucz 1993). Non-LTE lines (solid curves) have been calculated with SYNSPEC and TLUSTY (Hubeny \& Lanz 1995). In the upper panels the ratios between the two models are shown.

analysis. The temperature values of these two stars listed in Table 1 have to be regarded as lower limits.

The total estimated uncertainties including all the factors described above are given in Table 1.

\subsection{Mass loss}

For the stars showing mass loss effects in the $\mathrm{H}_{\alpha}$ line, wind emission and scattering were computed and added to the photospheric spectrum in the line fitting process, providing an estimate of the mass loss rate. The model we have adopted is based on the work developed initially by Panagia \& Felli (1975), Felli \& Panagia (1981), and Panagia (1991). Theoretical $\mathrm{H}_{\alpha}$ profiles were calculated with the procedure implemented by Scuderi et al. (1992) and by Scuderi et al. (1994) with the inclusion of the effect of the electron scattering on the $\mathrm{H}_{\alpha}$ profile. The wind is assumed to be spherically symmetric, stationary, and isothermal, and hydrogen to be fully ionized. The velocity field is expressed as a truncated power law: see Eqs. (1) and (2) of Scuderi et al. (1992). The radiative transfer is treated in the Sobolev approximation. The theoretical profiles are a combination of a photosperic profile with scattering and emission from hydrogen atoms and scattering from electrons in the wind. Therefore, the photospheric parameters $T_{\text {eff }}$ and $\log g$, and the mass loss rate, are derived concurrently with an iterative process.

The uncertainty on the mass loss rate was computed taking into account the errors in the fit of the emission profile and the uncertainties in $T_{\text {eff }}$ and $\log g$ (see Scuderi et al. 1992). 

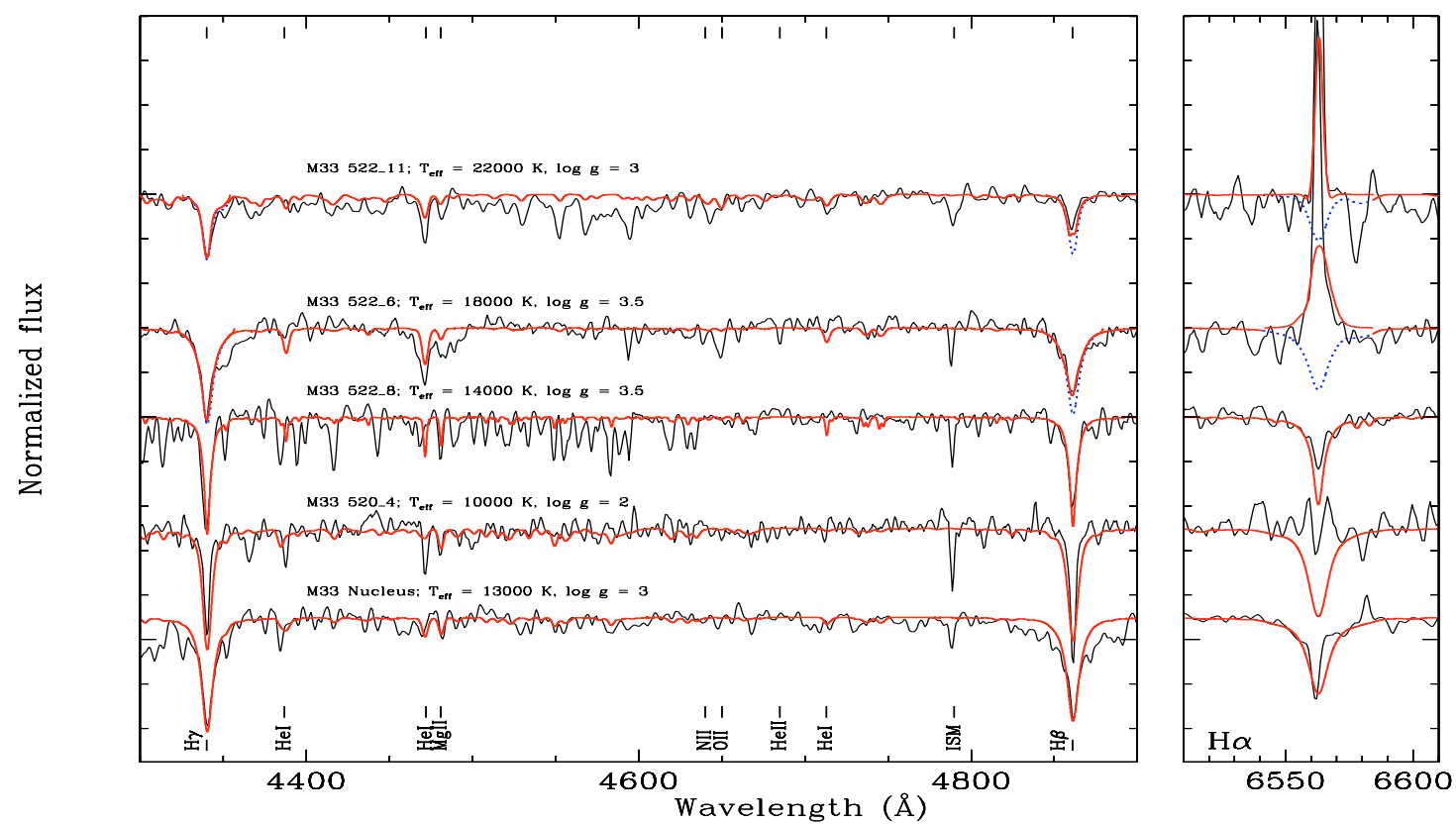

Fig. 4. Normalized spectra of stars observed in M 33. The smooth thick lines are the models for the adopted temperatures and gravities with an estimated wind emission, and the dashed lines refer to the photospheric models calculated before the mass-loss correction. All the spectra have been smoothed by a factor of three.
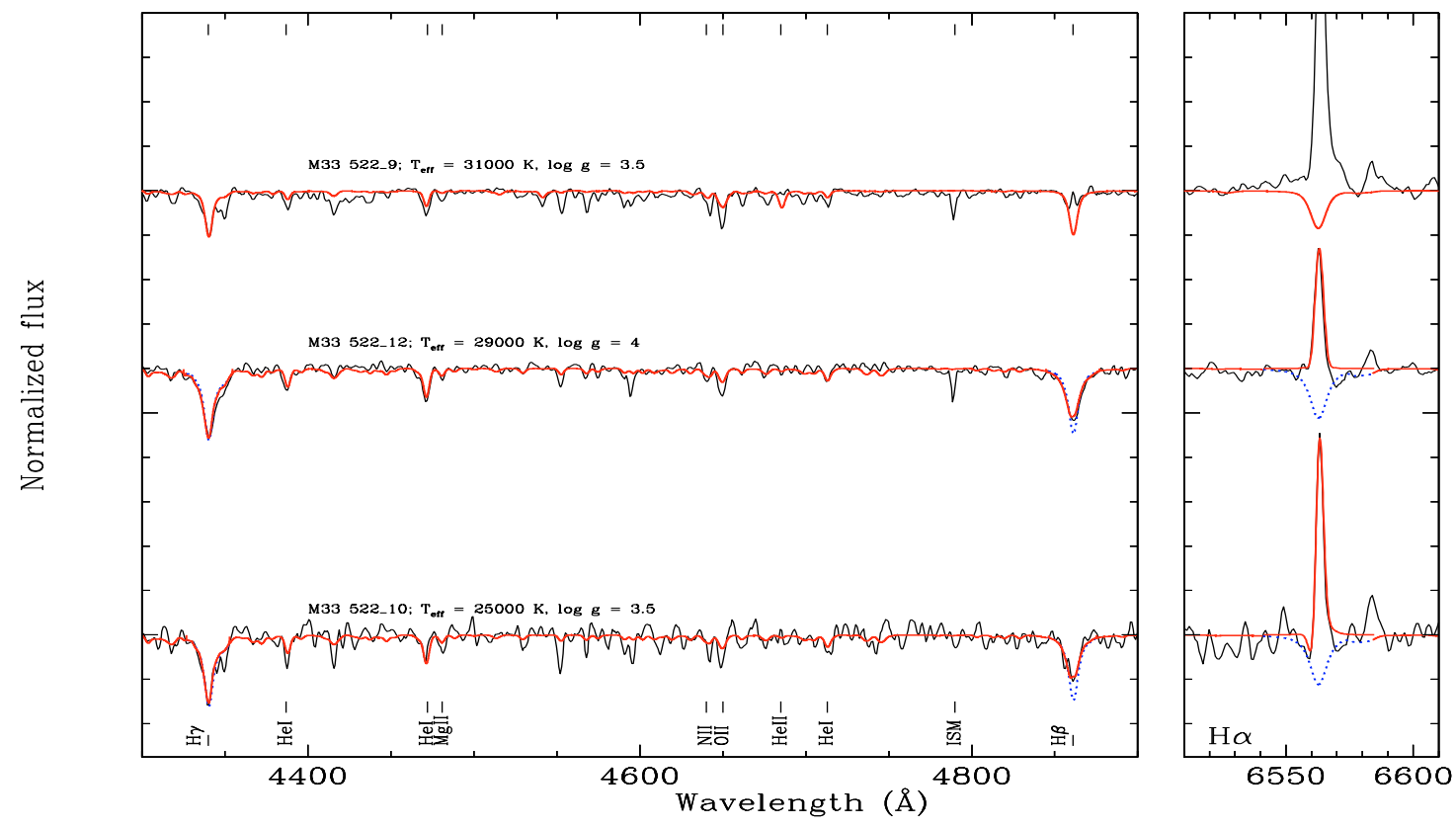

Fig. 5. Normalized spectra of stars observed in M33. Symbols are as in Fig. 4. For the middle and bottom stars the emission was fitted as if totally due to the stellar wind. However an unresolved nebular contribution may be present. For the top star, $\mathrm{H}_{\alpha}$ and $\mathrm{H}_{\beta}$ are totally filled by nebular emission.

For the stars whose spectra do not cover the $\mathrm{H}_{\alpha}$ region or for which the $\mathrm{H}_{\alpha}$ line is contaminated by nebular emission, we stopped our fitting procedure when we achieved a good match to the observed $\mathrm{H}_{\beta}$ and $\mathrm{H}_{\gamma}$ lines.

\section{Results}

From the M 33 data we analyzed seven serendipitous stars plus a spectrum of the galactic nucleus. The $S / N$ ratio of these spectra is sufficient to analyze a number of spectral features. Results from our model analysis are shown in Figs. 4-6 and the corresponding photosperic and wind parameters are listed in Table 1. Each figure shows the observed spectra in the range $4300-4900 \AA$ (to include $\mathrm{H}_{\gamma}$ and $\mathrm{H}_{\beta}$ ) and in a separate box the region around $\mathrm{H}_{\alpha}$ from the red spectra. The thick lines overimposed on every spectrum are the best fit synthetic model. When present, the dashed lines show the photospheric Balmer lines before the inclusion of mass-loss. 

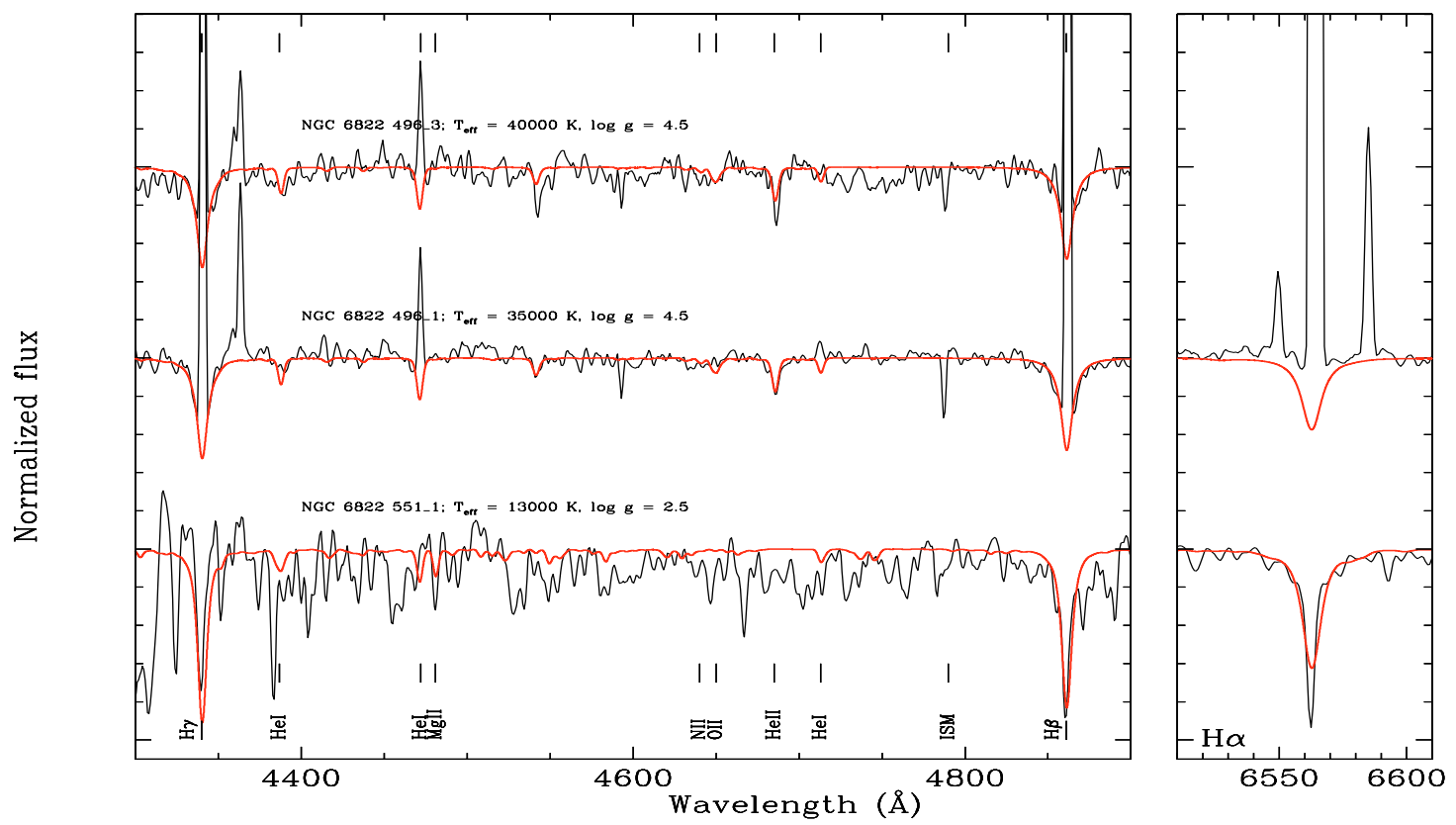

Fig. 6. Normalized spectra of the NGC 6822 stars. Symbols are as in Fig. 4. The severe contamination by nebular emission is evident in the two upper stars, which are in the Hubble V HII region.

For two stars in M33: 522_10 and 522_12 the models shown in Fig. 5 reproduce the observations quite well; both $\mathrm{H}$ (including $\mathrm{H}_{\alpha}$ ) and $\mathrm{HeI}$ lines are well fitted when mass-loss rates with the values indicated in Table 1 are applied. However, nebular emission is observed in the spectra very close to the stellar emission. Therefore, some unaccounted nebular contamination may still be present in the extracted spectra. If this is the case, then the values of the parameters in Table 1 should be regarded as upper limits.

For M 33 522_6 and M 33 520_11, $\mathrm{H}_{\alpha}$ is contaminated by nebular emission. Hence, only $\mathrm{H}_{\beta}$ and $\mathrm{H}_{\gamma}$ were used in the analysis, so the estimate of $\dot{M}$ is much more uncertain than for the case when the $\mathrm{H}_{\alpha}$ profile is available.

As for M 33 520_4 and 522_8, the $\mathrm{H}_{\alpha}$ absorptions are filled by nebular emission. In fact, no $\dot{M}$ correction was necessary to fit the blue lines in their spectra, consistent with their cool temperatures.

Finally, for M 33522.9 , the hottest star of our sample, only $\mathrm{H}_{\gamma}$ could be used in the analysis, both $\mathrm{H}_{\alpha}$ and $\mathrm{H}_{\beta}$ being masked by nebular emission. The photospheric parameters $T_{\text {eff }}$ and $\log g$ are consequently very uncertain.

In the NGC 6822 fields, we could extract only three serendipitous stars, but for one of these (NGC 6822551 1) the $S / N$ is very poor $(\approx 10)$ and the lines of the other two are strongly contaminated by nebular emission. Hence, we could not follow the procedure described for M 33 stars and we just overplot the synthetic models that matched at least the wing of the Balmer lines. Results are reported in Table 1 and displayed in Fig. 6.

\subsection{M33 nucleus}

The M 33 nucleus appears as a point-like source, van den Bergh (1991) describes it as "semistellar" with a luminosity higher than any galactic globular cluster. From the spectroscopic point of view, this author describes the nucleus as composite, with characteristics ranging from late A to F. Massey et al. (1996) found the spectrum of the nucleus similar to that of a supergiant of spectral type FIa. The M33's "bulge" was found to contain both a young, metal-rich population as well as older stars (Cowley et al. 1982; O’Connell 1983; Ciani et al. 1984; Schmidt et al. 1990). Comparing the spectrum of M33's nucleus with our single star models, the Balmer lines are clearly dominated by the A supergiant type population, although the overall spectrum of the nucleus is consistent with a composite population.

\section{Discussion and conclusion}

From their relative distances along the spectroscopic slit in the spatial direction, we identified the serendipitous stars in astrometrically calibrated images, where we measured their positions (given in Table 1).

As a consistency check for the temperatures and gravities derived from the spectroscopic analysis, we searched whether photometric observations of our stars exist. All our program stars in M 33 have photometric measurements by Massey et al. (1996). However, by inspecting recent higher resolution images (HST-WFPC 2 or ground-based, see Table 1 for details) we found that two stars (M $33522 \_$and M 33522 9) are in a compact cluster and the ground based photometry of Massey et al. (1996) probably include the whole cluster. M $33520 \_4$ is close to a star at $\alpha=01: 33: 40.30$ and $\delta=+30: 31: 59.0$ (OB12-21, Massey et al. 1995) which is fainter by about $0.8 \mathrm{mag}$ in the $V$ band. Our spectrum is not contaminated by the flux of the nearby object, but probably the photometry is. As for M33: 520_11 and 522_12 the ground-based photometry (Massey et al. 1996) indicates three other objects within 3 ", so the 
Table 1. List of the observed stars in M 33 and NGC 6822 with their physical properties. Stars in M 33 have been identified using the HST WFPC2 archive image root number U2TR03O1T (Massey) and the image for M 33 south downloaded from the web site of the Nearby Galaxies Survey Project (Massey et al. 2001). Stars in NGC 6822 have been identified using a VLT image with sub arcsec seeing (Bianchi 2002, priv. comm.) and with the HST WFPC2 images published by Bianchi et al. (2001b). The source for the photometry of the M33 objects is Massey et al. (1996) and for the NGC 6822 stars is Bianchi et al. (2001b).

\begin{tabular}{|c|c|c|c|c|c|c|c|c|}
\hline Star & $\begin{array}{l}\alpha \underset{(2000.0)}{ } \delta \\
\end{array}$ & $U$ & $B$ & $V$ & $\begin{array}{c}T_{\text {eff }} \\
\left(\times 10^{3} \mathrm{~K}\right)\end{array}$ & $\log g$ & $\begin{array}{c}\dot{M} \\
\left(10^{-7} M_{\odot} / \mathrm{yr}\right)\end{array}$ & Other ID \\
\hline M 33 Nucleus & $01: 33: 50.92+30: 39: 36.8$ & 14.94 & 14.58 & 14.03 & $(13.0 \pm 2.2)$ & $(3.0 \pm 0.2)$ & & \\
\hline M 33 520_4 $4^{a}$ & $01: 33: 40.54+30: 31: 58.5$ & 17.32 & 18.04 & 18.07 & $10.0 \pm 2.7$ & $2.0 \pm 0.3$ & & \\
\hline M 33 522_8 ${ }^{b}$ & $01: 33: 39.29+30: 31: 25.8$ & 17.69 & 18.49 & 18.65 & $14.0 \pm 2.6$ & $3.5 \pm 0.3$ & & OB12-13 \\
\hline M 33 522_6 & $01: 33: 39.83+30: 31: 36.3$ & 17.19 & 18.04 & 18.26 & $18.0 \pm 2.6$ & $3.5 \pm 0.3$ & $3.4 \pm 2.0$ & OB12-17 \\
\hline M 33 520_11 ${ }^{b}$ & $01: 33: 39.13+30: 31: 18.3$ & 17.62 & 18.52 & 16.85 & $22.0 \pm 2.7$ & $3.0 \pm 0.3$ & $1.5 \pm 1.0:$ & \\
\hline M 33 522_10 & $01: 33: 39.20+30: 31: 21.1$ & 17.61 & 18.50 & 18.73 & $25.0 \pm 2.4:$ & $3.5 \pm 0.3:$ & $4.2 \pm 2.1$ & \\
\hline M 33 522_12 & $01: 33: 39.01+30: 31: 14.8$ & 16.62 & 17.44 & 17.63 & $29.0 \pm 2.0:$ & $4.0 \pm 0.4:$ & $2.1 \pm 1.0$ & OB12-12 \\
\hline M 33 522_. $9^{b}$ & $01: 33: 39.29+30: 31: 24.1$ & 15.99 & 16.90 & 17.03 & $31.0 \pm 3.0:$ & $<3.5$ & & OB12-14 \\
\hline NGC 6822 551_1 & $19: 45: 15.55-14: 43: 11.1$ & 16.95 & 17.69 & 17.52 & $13.0 \pm 4.0$ & $2.5 \pm 0.3$ & & CTIO-695 \\
\hline NGC 6822 496_1 ${ }^{c}$ & $19: 45: 05.20-14: 43: 17.2$ & 18.78 & 19.70 & 19.64 & $>30.0$ & - & & LB-f2-977 \\
\hline NGC $6822496 \_3^{d}$ & $19: 45: 05.34-14: 43: 14.8$ & 18.65 & 19.58 & 19.57 & $>30.0$ & - & & LB-f2-999 \\
\hline
\end{tabular}

${ }^{a}$ Photometry is probably contaminated by a nearby object at $\alpha=01: 33: 40.30$ and $\delta=+30: 31: 59.0$ (Massey et al. 1995, OB12-21).

${ }^{b}$ HST imaging shows that these stars are in a cluster, ground-based photometric measurements are contaminated.

${ }^{c}$ This star is in the Hubble V region, within 2" there are other two stars with magnitudes $V=20.23$ and $V=22.08$ (from the HST photometry of Bianchi et al. (2001b).

${ }^{d}$ This star is in the Hubble V region, within 2" there are other six stars with $V$ magnitudes about 20 (from the HST photometry of Bianchi et al. (2001b).

:) results are uncertain due to nebular emission.

photometric colors reported in Table 1 are probably contaminated. We were able to identify these stars in high resolution images (see Table 1), for which photometry is not yet available.

In summary, the existing photometry cannot be used as a reliable luminosity check as our targets lie in particularly crowded clusters. Further interpretation must await better photometric measurements to become available.

From the spectroscopic point of view, the $S / N$ of our spectra did not allow a precise abundance analysis since most of the weak lines are confused at noise level in our sample. However, for two stars displayed in Fig. 5: M33 $522 \_12$ and M $33522 \_9$, which have $S / N$ is $\approx 50$, our models reproduce also the MgII 4481.150, NII 4641.028 and OII $4650.103 \AA$ lines. The latter two seem to reflect an overabundance of such elements with respect the value of $Z$ used in our modeling, but higher resolution and higher $S / N$ spectra are necessary to support this conclusion.

The winds of hot stars are driven by photon momentum transfer through metal line absorption so it is obvious that the global properties of the winds must be a function of stellar metallicity (Abbott 1982). Comparison of derived wind parameters of $\mathrm{O}$ stars in the Galaxy and in the Magellanic Clouds shows an indication, more evident for the SMC than LMC, of metallicity dependence (Kudritzki \& Puls 2000).

M 33 metallicity is on average similar to that of the LMC. Thus, according to Abbott (1982) mass-loss rates measured for the stars of this galaxy should be lower than their Milky Way counterparts. Galactic B supergiants in this range of temperatures have mass loss rates of the order of $10^{-6} M_{\odot} \mathrm{yr}^{-1}$ (see for example Scuderi et al. 1998). All our objects have estimated mass-loss rates lower than this value up to one order of magnitude, which is what one would expect from a lower metallicity star. However no definitive conclusion can be drawn with this respect because three out of four stars have gravities too high to be classified as supergiants so their lower luminosity can be the cause for the lower mass loss rate.

For the stars in NGC 6822, the limited quality of our spectra provides only a rough estimate of $T_{\text {eff for one object (which }}$ may be a foreground star), and an indication that two other objects may be very hot massive stars. Photometry and positions are based on HST-WFPC2 images (Bianchi et al. 2001b), and the identification of the spectroscopic stars was unambiguous. Furthermore, at temperatures of the order of $30000 \mathrm{~K}$, nonLTE effects are significant, hence our analysis only provides lower limits.

In summary, in M33 we found 5 hot star candidates out of 7 serendipitous stars analyzed, and 2 out of 3 in NGC 6822. Although the limited data quality does not afford a precise measurement of stellar parameters, our results indicate that the fields sample very rich OB associations. To our knowledge, none of the stars has previously published spectroscopy, except for M 33 522_12 (OB12-12, Massey et al. 1995) and the M 33 nucleus (Massey et al. 1996).

Thus, our spectra add new data-points to the census of hot massive stars in these nearby Local Group galaxies and contribute to the mass-loss rate determination in external galaxies for which, besides its importance, a few measurements have been so far available in literature. 
Acknowledgements. We are extremely grateful to Dr. Emmanuel Vassiliadis who participated in the observations at La Palma. LB and GC acknowledge support from NASA grant NAG5-9219 (NRA-9901-LTSA-029). GC is grateful to Prof. S. Catalano, director of the Catania Astrophysical Observatory, and to LB for support of his visit to JHU for completion of this work. Thanks are due to the referee, Henny Lamers, for his useful comments that helped us to improve the manuscript.

\section{References}

Abbott, D. L. 1982, ApJ, 259, 282

Bianchi, L., et al. 2003, in preparation

Bianchi, L., Catanzaro, G., Scuderi, S., \& Hutchings, J. B. 2001a, PASP, 113, 697, Paper I

Bianchi, L., Scuderi, S., Massey, P., \& Romaniello, M. 2001b, AJ, 121,2020

Chandar, R., Bianchi, L., \& Ford, H. 2000, AJ, 120, 3088

Ciani, A., D’Odorico, S., \& Benvenuti, P. 1984, A\&A, 137, 223

Cowley, A. P., Crampton, D., \& McClure, R. D. 1982, ApJ, 263, 1

Felli, M., \& Panagia, N. 1981, A\&A, 102, 424

Garmany, C., \& Conti, P. S. 1985, ApJ, 293, 407

Gray, D. F. 1976, The observation and analysis of stellar photosperes, Cambridge Astrophysics Ser., 386

Hubeny, I., \& Lanz, T. 1995, ApJ, 439, 875

Hutchings, J. B. 1982, ApJ, 255, 70

Kudritzki, R. P., \& Puls, J. 2000, ARA\&A, 38, 613
Kurucz, R. L. 1993, in IAU Col. 138, Peculiar versus normal phenomena in A-type and related stars, ed. M. M. Dworetsky, F. Castelli, $\&$ R. Faraggiana, ASP Conf. Ser., 44, 87

Kurucz, R. L., \& Avrett, E. H. 1981, SAO Special Report 391

Massey, P., Armandroff, T. E., Pyke, R., Patel, K., \& Wilson, C. D. 1995, AJ, 110, 2715

Massey, P., Bianchi, L., Hutchings, J. B., \& Stecher, T. P. 1996, ApJ, 469,629

Massey, P., Hodge, P. W., Holmes, S., et al. 2001, AAS, 199, 130.05

Muschielok, B., Kudritzki, R. P., Appenzeller, I., et al. 1999, A\&A, 352, L40

O'Connel, R. W. 1983, ApJ, 267, 80

Pagel, B. E. J., Edmunds, M. G., \& Smith, G. 1980, MNRAS, 193, 219

Panagia, N. 1991, in The physics of star formation and early star evolution, ed. C. J. Lada, \& N. D. Kylafis (Kluwer Academic Publishers), 565

Panagia, N., \& Felli, M. 1975, A\&A, 39, 1

Russell, S. C., \& Dopita, M. A. 1990, ApJS, 74, 93

Schmidt, A. A., Bica, E., \& Alloin, D. 1990, MNRAS, 243, 620

Scuderi, S., Bonanno, G., Di Benedetto, R., Spadaro, D., \& Panagia, N. 1992, ApJ, 329, 201

Scuderi, S., Bonanno, G., Spadaro, et al. 1994, ApJ, 437, 465

Scuderi, S., Panagia, N., Stanghellini, C., Trigilio, C., \& Umana, G. 1998, A\&A, 332, 251

Vilchez, J. M., Pagel, B. E. J., Diaz, A. I., Terlevich, E., \& Edmunds, M. G. 1988, MNRAS, 235, 633

van den Bergh, S. 1991, PASP, 103, 609 